\title{
PROBABILISTIC ANALYSIS OF THE TELEGRAPHER'S PROCESS WITH DRIFT BY MEANS OF RELATIVISTIC TRANSFORMATIONS
}

\author{
L. BEGHIN, L. NIEDDU and E. ORSINGHER \\ University of Rome "La Sapienza" \\ Dipartimento di Statistica, Probabilità e Statistiche Applicate \\ Rome, Italy
}

(Received December, 1999; Revised June, 2000)

\begin{abstract}
The telegrapher's process with drift is here examined and its distribution is obtained by applying the Lorentz transformation. The related characteristic function as well as the distribution are also derived by solving an initial value problem for the generalized telegraph equation.

Key words: Telegraph Equation, Relativistic Transformations, Bessel Functions, Flow Functions, Fourier Transform.
\end{abstract}

AMS subject classifications: $60 \mathrm{~K} 99,60 \mathrm{H} 30$.

\section{Introduction}

In this paper we consider the two-valued integrated telegraph signal with rightward velocity $c_{1}$ and leftward velocity $-c_{2}\left(c_{1}, c_{2}>0\right)$ and rates $\lambda_{1}, \lambda_{2}$ of the occurrence of velocity switches, $\left(\lambda_{i}\right.$ when the current velocity is $\left.(-1)^{i+1} c_{i}, i=1,2\right)$.

The classical case $\left(c_{1}=c_{2}=c ; \lambda_{1}=\lambda_{2}=\lambda\right)$ has been studied in many papers and important probabilistic distributions and representations have been obtained independently by various authors and by different methods (for example, Orsingher [8], Foong [2], Foong and Kanno [3], Kabanov [4]).

When $c_{1} \neq c_{2}$ and $\lambda_{1} \neq \lambda_{2}$, the motion differs from that in the classical case in that it displays a drift whose components have also been studied (see $[1,6,7]$ ). One component of the drift depends on the different velocities and the other on the different rates. These components differ substantially in the mathematical treatment they necessitate.

In particular, when $\lambda_{1} \neq \lambda_{2}$, the elimination of the drift requires the Lorentz transformation of Special Relativity Theory. This was first noted by Cane [1] and further examined in $[6,7]$ but nowhere has an accurate analysis of the transformation and its probabilistic implications been carried out.

Here we discuss the random motion in the original frame of reference $(x, t)$ and in the related relativistic one, $\left(x^{\prime}, t^{\prime}\right)$ where the drift has been eliminated. 
The space coordinate $x^{\prime}$ must move with velocity $v_{r}=\frac{c_{1}-c_{2}}{2}+$ $\frac{\left(\lambda_{2}-\lambda_{1}\right)\left(c_{1}+c_{2}\right)}{2\left(\lambda_{2}+\lambda_{1}\right)}=\frac{\lambda_{2} c_{1}-\lambda_{1} c_{2}}{\lambda_{1}+\lambda_{2}}$ with respect to the original frame of reference and the time $t^{\prime}$ must either be speeded up or slowed down with respect to $t$, in order to eliminate the drift.

In the frame $\left(x^{\prime}, t^{\prime}\right)$, the particle moves with velocities $c^{\prime}= \pm \frac{2\left(c_{1}+c_{2}\right) \lambda_{1} \lambda_{2}}{\left(\lambda_{1}+\lambda_{2}\right)^{2}}$, initially chosen with equal probability $1 / 2$, and the switches from positive to negative values and vice versa are governed by a homogeneous Poisson process with rate $\lambda^{\prime}=$ $\frac{2 \lambda_{1} \lambda_{2}}{\lambda_{1}+\lambda_{2}}$

Therefore, the probabilist, in the reference $\left(x^{\prime}, t^{\prime}\right)$ attributes to the random position of the particle, a symmetric distribution $p=p\left(x^{\prime}, t^{\prime}\right)$. Returning to the original coordinates and writing down the asymmetric distribution $p=p(x, t)$ requires careful attention due to the fact that here, differently from the Special Relativity theory, the adjustment of time depends on the random changes of the rates (and thus of the velocity of the particle).

In the last section of the paper we obtain the distribution $p=p(x, t)$ by means of the usual approach, based on Fourier transforms. This also enables us to present the characteristic function in the case where a general form of drift is assumed.

The reader can easily judge how significant the simpification using the relativistic transformation is and how deep an insight into the intimate structure of the random motion is afforded.

The relativistic approach also immediately yields the form of the flow function and therefore the joint distributions of the position and of the velocity of the particle.

\section{Features of Motion and the Governing Equation}

We assume that at time $t=0$, a particle starts from the origin and that its initial velocity is the two-valued r.v.

$$
V(0)= \begin{cases}c_{1} & \text { with probability } \frac{1}{2} \\ -c_{2} & \text { with probability } \frac{1}{2}\end{cases}
$$

where $c_{1}, c_{2}$ are positive, real numbers.

The current velocity $V=V(t), t>0$ switches from $c_{1}$ to $-c_{2}$ after an exponentially distributed time (with parameter $\lambda_{1}$ ) and from $-c_{2}$ to $c_{1}$ after a random time with exponential distribution with parameter $\lambda_{2}$.

The time intervals separated by velocity changes are independent r.v.s (also independent from $V(0))$.

Thus the particle moves forward with velocity $c_{1}$ and backward with velocity $-c_{2}$ and the changes are governed by a non-homogeneous Poisson process.

For the probabilistic description of the random position $X=X(t)=\int{ }_{0}^{t} V(s) d s$ we need the following distributions

$$
\left\{\begin{array}{c}
f_{1}(x, t) d x=\operatorname{Pr}\left\{X(t) \in d x, V(t)=c_{1}\right\} \\
f_{2}(x, t) d x=\operatorname{Pr}\left\{X(t) \in d x, V(t)=-c_{2}\right\} .
\end{array}\right.
$$


It is well known that the functions (2.1) are solutions of the following differential system (see [5])

$$
\left\{\begin{array}{c}
\frac{\partial f_{1}}{\partial t}=-c_{1} \frac{\partial f_{1}}{\partial x}+\lambda_{2} f_{2}-\lambda_{1} f_{1} \\
\frac{\partial f_{2}}{\partial t}=c_{2} \frac{\partial f_{2}}{\partial x}+\lambda_{1} f_{1}-\lambda_{2} f_{2}
\end{array}\right.
$$

The system (2.2) by means of the transformation

$$
p=f_{1}+f_{2}, w=f_{1}-f_{2}
$$

can equivalently be written down as

$$
\left\{\begin{array}{c}
\frac{\partial p}{\partial t}=-\frac{c_{1}-c_{2}}{2} \frac{\partial p}{\partial x}-\frac{c_{1}+c_{2}}{2} \frac{\partial w}{\partial x} \\
\frac{\partial w}{\partial t}=-\frac{c_{1}+c_{2}}{2} \frac{\partial p}{\partial x}-\frac{c_{1}-c_{2}}{2} \frac{\partial w}{\partial x}-\left(\lambda_{1}-\lambda_{2}\right) p-\left(\lambda_{1}+\lambda_{2}\right) w
\end{array}\right.
$$

The distribution $p(x, t) d x=\operatorname{Pr}\{X(t) \in d x\}$ consists of a singular component concentrated in $x=c_{1} t$ (with probability $\frac{1}{2} e^{-\lambda_{1} t}$ ) and in $x=-c_{2} t$ (with probability $\left.\frac{1}{2} e^{-\lambda_{2} t}\right)$ and an absolutely continuous part spread over the interval $\left(-c_{2} t, c_{1} t\right)$.

The absolutely continuous part of the distribution is a solution of the second-order hyperbolic equation (extracted from the differential system (2.3) by means of subsequent differentiations and substitutions):

$$
\begin{aligned}
& \frac{\partial^{2} p}{\partial t^{2}}=c_{1} c_{2} \frac{\partial^{2} p}{\partial x^{2}}+\left(c_{2}-c_{1}\right) \frac{\partial^{2} p}{\partial x \partial t}-\left(\lambda_{1}+\lambda_{2}\right) \frac{\partial p}{\partial t} \\
& +\frac{1}{2}\left[\left(c_{2}-c_{1}\right)\left(\lambda_{1}+\lambda_{2}\right)-\left(\lambda_{2}-\lambda_{1}\right)\left(c_{1}+c_{2}\right)\right] \frac{\partial p}{\partial x} .
\end{aligned}
$$

The presence of $\frac{\partial p}{\partial x}$ and $\frac{\partial^{2} p}{\partial x \partial t}$ in (2.4) is clearly related to the drift of motion.

Equation (2.4), when $c_{1}=c_{2}=c$ and $\lambda_{1}=\lambda_{2}=\lambda$, reduces to the classical telegraph equation

$$
\frac{\partial^{2} p}{\partial t^{2}}=c^{2} \frac{\partial^{2} p}{\partial x^{2}}-2 \lambda \frac{\partial p}{\partial t}
$$

\section{Elimination of the Drift by Means of a Relativistic Transformation}

The elimination of the drift necessitates the use of the Lorentz transformation

$$
\left\{\begin{array}{l}
x^{\prime}=\alpha x+\beta t \\
t^{\prime}=\gamma x+\delta t
\end{array}\right.
$$

where the constants $\alpha, \beta, \gamma, \delta$ are to be determined in such a way that the coefficients of $\frac{\partial^{2} p}{\partial x^{\prime} \partial t^{\prime}}$ and $\frac{\partial p}{\partial x^{\prime}}$, vanish. In order to evaluate the four parameters in (3.1), clearly, 
two further conditions must be introduced.

We now have our first theorem.

Theorem 3.1: A linear transformation from the frame of reference $(x, t)$ into $\left(x^{\prime}, t^{\prime}\right)$, capable of eliminating the drift in (2.4) is

$$
\left\{\begin{array}{c}
x^{\prime}=x+\frac{\left(c_{2}-c_{1}\right)\left(\lambda_{2}+\lambda_{1}\right)-\left(\lambda_{2}-\lambda_{1}\right)\left(c_{1}+c_{2}\right)}{2\left(\lambda_{1}+\lambda_{2}\right)} t \\
t^{\prime}=-\frac{\left(\lambda_{2}^{2}-\lambda_{1}^{2}\right)}{2 \lambda_{1} \lambda_{2}\left(c_{1}+c_{2}\right)} x+\left\{\frac{\left(\lambda_{2}+\lambda_{1}\right)^{2}}{4 \lambda_{1} \lambda_{2}}-\frac{\left(c_{2}-c_{1}\right)}{4 \lambda_{1} \lambda_{2}} \frac{\left(\lambda_{2}^{2}-\lambda_{1}^{2}\right)}{\left(c_{2}+c_{1}\right)}\right\} t
\end{array}\right.
$$

Proof: We first remark that any transformation of the form

$$
\left\{\begin{array}{l}
x^{\prime}=\alpha\left\{x+\frac{\left(c_{2}-c_{1}\right)\left(\lambda_{1}+\lambda_{2}\right)-\left(\lambda_{2}-\lambda_{1}\right)\left(c_{1}+c_{2}\right)}{2\left(\lambda_{2}+\lambda_{1}\right)} t\right\} \\
t^{\prime}=\gamma\left\{x+\frac{\left(\lambda_{2}-\lambda_{1}\right)\left(c_{2}^{2}-c_{1}^{2}\right)-\left(\lambda_{2}+\lambda_{1}\right)\left(c_{1}+c_{2}\right)^{2}}{2\left(\lambda_{2}-\lambda_{1}\right)\left(c_{1}+c_{2}\right)} t\right\}
\end{array}\right.
$$

eliminates the coefficients of $\frac{\partial p}{\partial x^{\prime}}$ and $\frac{\partial^{2} p}{\partial x^{\prime} \partial t^{\prime}}$.

Assuming that $\alpha=1$ and that the Jacobian of (3.3) is equal to 1 , we get that

$$
\gamma=-\frac{\lambda_{2}^{2}-\lambda_{1}^{2}}{2 \lambda_{1} \lambda_{2}\left(c_{1}+c_{2}\right)}
$$

This completes the proof of (3.2).

Theorem 3.2: In force of the Lorentz transformation (3.2), equation (3.2) is converted to the following telegraph equation with respect to the space-time coordinates $\left(x^{\prime}, t^{\prime}\right)$ :

$$
\frac{\partial^{2} p}{\partial t^{\prime 2}}=\frac{4\left(c_{1}+c_{2}\right)^{2} \lambda_{1}^{2} \lambda_{2}^{2}}{\left(\lambda_{1}+\lambda_{2}\right)^{4}} \frac{\partial^{2} p}{\partial x^{\prime 2}}-\frac{4 \lambda_{1} \lambda_{2}}{\lambda_{1}+\lambda_{2}} \frac{\partial p}{\partial t^{\prime}} .
$$

Proof: We first observe that in the frame $\left(x^{\prime}, t^{\prime}\right)$, after the elimination of the drift, equation (2.4) is transformed into the following one

$$
\begin{gathered}
\left\{\delta^{2}-c_{1} c_{2} \gamma^{2}-\gamma \delta\left(c_{2}-c_{1}\right)\right\} \frac{\partial^{2} p}{\partial t^{\prime 2}}=\left\{\alpha c_{1} c_{2}-\beta^{2}+\alpha \beta\left(c_{2}-c_{1}\right)\right\} \frac{\partial^{2} p}{\partial x^{\prime 2}} \\
+\left\{\frac{\delta}{2}\left(\left(c_{2}-c_{1}\right)\left(\lambda_{1}+\lambda_{2}\right)-\left(\lambda_{2}-\lambda_{1}\right)\left(c_{1}+c_{2}\right)\right)-\left(\lambda_{1}+\lambda_{2}\right) \delta\right\} \frac{\partial p}{\partial t^{\prime}} .
\end{gathered}
$$

In light of (3.2) we have that

$$
\left\{\begin{array}{c}
\alpha=1 \\
\gamma=\frac{\left(\lambda_{2}^{2}-\lambda_{1}^{2}\right)}{2 \lambda_{1} \lambda_{2}\left(c_{1}+c_{2}\right)} \\
\beta=\frac{c_{2}-c_{1}}{2}-\frac{\left(\lambda_{2}-\lambda_{1}\right)}{\left(\lambda_{2}+\lambda_{1}\right)} \frac{\left(c_{2}+c_{1}\right)}{2} \\
\delta=\frac{\left(\lambda_{1}+\lambda_{2}\right)^{2}}{4 \lambda_{1} \lambda_{2}}-\frac{\left(\lambda_{2}^{2}-\lambda_{1}^{2}\right)^{2}\left(c_{2}-c_{1}\right)}{4 \lambda_{1} \lambda_{2}\left(c_{1}+c_{2}\right)}
\end{array}\right.
$$


and therefore

$$
\left\{\begin{array}{c}
\delta^{2}-c_{1} c_{2} \gamma^{2}-\gamma \delta\left(c_{2}-c_{1}\right)=\frac{\left(\lambda_{1}+\lambda_{2}\right)^{2}}{4 \lambda_{1} \lambda_{2}} \\
\alpha c_{1} c_{2}-\beta^{2}+\alpha \beta\left(c_{2}-c_{1}\right)=\frac{\left(c_{1}+c_{2}\right)^{2}}{4}\left\{1-\frac{\left(\lambda_{2}-\lambda_{1}\right)^{2}}{\left(\lambda_{2}+\lambda_{1}\right)^{2}}\right\} \\
\frac{\delta}{2}\left\{\left(c_{1}-c_{1}\right)\left(\lambda_{1}+\lambda_{2}\right)-\left(\lambda_{2}-\lambda_{1}\right)\left(c_{1}+c_{2}\right)\right\}-\delta\left(\lambda_{1}+\lambda_{2}\right)=-\left(\lambda_{1}+\lambda_{2}\right) .
\end{array}\right.
$$

Substituting (3.7) into (3.5) and then dividing by $\frac{\left(\lambda_{1}+\lambda_{2}\right)^{2}}{4 \lambda_{1} \lambda_{2}}$ we readily obtain equa-

Remark 3.1: We are now able to infer from equation (3.4) some important features of the random motion in the frame $\left(x^{\prime}, t^{\prime}\right)$.

For an observer in this system (compare equation (3.4) with (2.5)) of reference, the particle moves with velocities

$$
c^{\prime}= \pm \frac{2\left(c_{1}+c_{2}\right) \lambda_{1} \lambda_{2}}{\left(\lambda_{1}+\lambda_{2}\right)^{2}}
$$

and the switches between the two values (3.8) are governed by a homogeneous Poisson process whose rate is

$$
\lambda^{\prime}=\frac{2 \lambda_{1} \lambda_{2}}{\lambda_{1}+\lambda_{2}}
$$

Remark 3.2: The connection between the velocities in $(x, t)$ and in $\left(x^{\prime}, t^{\prime}\right)$ is given by the formula

$$
\frac{d x^{\prime}}{d t^{\prime}}=\frac{\frac{d x}{d t}+\frac{\left(c_{2}-c_{1}\right)}{2}-\frac{\left(\lambda_{2}-\lambda_{1}\right)\left(c_{2}+c_{1}\right)}{2\left(\lambda_{1}+\lambda_{2}\right)}}{-\frac{\left(\lambda_{2}^{2}-\lambda_{1}^{2}\right)}{2 \lambda_{1} \lambda_{2}\left(c_{1}+c_{2}\right)} \frac{d x}{d t}+\frac{\left(\lambda_{1}+\lambda_{2}\right)^{2}}{4 \lambda_{1} \lambda_{2}}-\frac{\left(c_{2}-c_{1}\right)\left(\lambda_{2}^{2}-\lambda_{1}^{2}\right)}{4 \lambda_{1} \lambda_{2}\left(c_{1}+c_{2}\right)}}
$$

which can be straightforwardly obtained from (3.2).

From (3.10) it is easy to see that

$$
\text { if } \frac{d x}{d t}=c_{1} \text {, then } \frac{d x^{\prime}}{d t^{\prime}}=c^{\prime}=\frac{2\left(c_{1}+c_{2}\right) \lambda_{1} \lambda_{2}}{\left(\lambda_{1}+\lambda_{2}\right)^{2}}
$$

and

$$
\text { if } \frac{d x}{d t}=-c_{2} \text {, then } \frac{d x^{\prime}}{d t^{\prime}}=c^{\prime}=-\frac{2\left(c_{1}+c_{2}\right) \lambda_{1} \lambda_{2}}{\left(\lambda_{1}+\lambda_{2}\right)^{2}} \text {. }
$$

Remark 3.3: From (3.2) it is also possible to extract the relationship

$$
\frac{d t^{\prime}}{d t}=-\frac{\lambda_{2}^{2}-\lambda_{1}^{2}}{2 \lambda_{1} \lambda_{2}\left(c_{1}+c_{2}\right)} \frac{d x}{d t}+\frac{\left(\lambda_{1}+\lambda_{2}\right)^{2}}{4 \lambda_{1} \lambda_{2}}-\frac{c_{2}-c_{1}}{c_{2}+c_{1}} \frac{\lambda_{2}^{2}-\lambda_{1}^{2}}{4 \lambda_{1} \lambda_{2}}
$$

which tells us how the time $t^{\prime}$ changes with $t$ (as a function of the velocity $\frac{d x}{d t}$ ). We have the following picture: 


$$
\frac{d t^{\prime}}{d t}= \begin{cases}\frac{\lambda_{1}+\lambda_{2}}{2 \lambda_{2}} & \text { if } \frac{d x}{d t}=c_{1} \\ \frac{\lambda_{1}+\lambda_{2}}{2 \lambda_{1}} & \text { if } \frac{d x}{d t}=-c_{2} .\end{cases}
$$

Formula (3.12) shows that the times $t^{\prime}$ and $t$ grow at the same rate if $\lambda_{1}=\lambda_{2}$.

This explains the fact that the part of the drift due to different rates must be canceled by suitably speeding up (or slowing down) the clock in $\left(x^{\prime}, t^{\prime}\right)$. For example, if $\lambda_{1}>\lambda_{2}$ (and the current velocity is $c_{1}$ in $(x, t)$ and $c^{\prime}$ in $\left(x^{\prime}, t^{\prime}\right)$ ) the time $t^{\prime}$ must be speeded up in order to compensate for the fact that switches from $c_{1}$ to $c_{2}$ occur more frequently then those from $c_{2}$ to $c_{1}$.

Remark 3.4: The connection between the interval of possible positions in $(x, t)$ and in $\left(x^{\prime}, t^{\prime}\right)$ can be discussed observing that:

$$
x^{\prime 2}-c^{\prime 2} t^{\prime 2}=\left\{1-\left(\frac{\lambda_{2}-\lambda_{1}}{\lambda_{2}+\lambda_{1}}\right)^{2}\right\}\left(x-c_{1} t\right)\left(x+c_{2} t\right) .
$$

Remark 3.5: In the frame $\left(x^{\prime}, t^{\prime}\right)$, the velocity $\left|c^{\prime}\right|$ is always inferior to the mean of the velocities $c_{1}$ and $c_{2}$. In fact we have, from (3.8), that

because $2 \lambda_{1} \lambda_{2} \leq \lambda_{1}^{2}+\lambda_{2}^{2}$.

$$
\left|c^{\prime}\right|=\frac{c_{1}+c_{2}}{2} \frac{4 \lambda_{1} \lambda_{2}}{\lambda_{1}^{2}+\lambda_{2}^{2}+2 \lambda_{1} \lambda_{2}} \leq \frac{c_{1}+c_{2}}{2}
$$

Remark 3.6: If $\lambda_{1}=\lambda_{2}=\lambda, c_{2} \neq c_{1}$, the transformation (3.2) reduces to the Galilean form

$$
\left\{\begin{array}{c}
x^{\prime}=x+\frac{\left(c_{2}-c_{1}\right)}{2} t \\
t^{\prime}=t
\end{array}\right.
$$

Thus the relative velocity $v_{r}=\frac{c_{1}-c_{2}}{2}$ and in $\left(x^{\prime}, t\right)$ the particle oscillates with velocities $c^{\prime}= \pm \frac{c_{1}+c_{2}}{2}$.

\section{The Distribution of the Position in the Frame $(x, t)$}

The Lorentz transformation discussed in Section 3 permits us to derive the distribution

$$
p(x, t) d x=\operatorname{Pr}\{X(t) \in d x\}
$$

from that of $p=p\left(x^{\prime}, t^{\prime}\right)$. By exploiting well known results in literature (see $[3,8]$ ) we can express $p\left(x^{\prime}, t^{\prime}\right)$ as follows:

$$
\begin{aligned}
p\left(x^{\prime}, t^{\prime}\right)= & \frac{e^{-\frac{2 \lambda_{1} \lambda_{2}}{\lambda_{1}+\lambda_{2}} t^{\prime}}}{\frac{4\left(c_{1}+c_{2}\right) \lambda_{1} \lambda_{2}}{\left(\lambda_{1}+\lambda_{2}\right)^{2}}}\left[\frac{2 \lambda_{1} \lambda_{2}}{\lambda_{1}+\lambda_{2}} I 0\left(\frac{\lambda_{1}+\lambda_{2}}{c_{1}+c_{2}} \sqrt{\frac{4\left(c_{1}+c_{2}\right)^{2} \lambda_{1}^{2} \lambda_{2}^{2} t^{\prime 2}}{\left(\lambda_{1}+\lambda_{2}\right)^{4}}-x^{\prime 2}}\right)\right. \\
& \left.+\frac{\partial}{\partial t^{\prime}} I 0\left(\frac{\lambda_{1}+\lambda_{2}}{c_{1}+c_{2}} \sqrt{\frac{4\left(c_{1}+c_{2}\right)^{2} \lambda_{1}^{2} \lambda_{2}^{2} t^{\prime 2}}{\left(\lambda_{1}+\lambda_{2}\right)^{4}}-x^{\prime 2}}\right)\right]
\end{aligned}
$$




$$
+\frac{e^{-\frac{2 \lambda_{1} \lambda_{2}}{\lambda_{1}+\lambda_{2}} t^{\prime}}}{2}\left\{\delta\left(x^{\prime}+\frac{2\left(c_{1}+c_{2}\right) \lambda_{1} \lambda_{2}}{\left(\lambda_{1}+\lambda_{2}\right)^{2}} t^{\prime}\right)+\delta\left(x^{\prime}-\frac{2\left(c_{1}+c_{2}\right) \lambda_{1} \lambda_{2} t^{\prime}}{\left(\lambda_{1}+\lambda_{2}\right)^{2}}\right)\right\}
$$

for $\left|x^{\prime}\right| \leq \frac{2\left(c_{1}+c_{2}\right) \lambda_{1} \lambda_{2} t^{\prime}}{\left(\lambda_{1}+\lambda_{2}\right)^{2}}$

Clearly

$$
I_{0}(x)=\sum_{k=0}^{\infty}\left(\frac{x}{2}\right)^{2 k} \frac{1}{(k !)^{2}}
$$

is the zero-order Bessel function with imaginary argument. In view of a result in [8] we can also write the expression for the flow function $w=w\left(x^{\prime}, t^{\prime}\right)$ in the following way

$$
w\left(x^{\prime}, t^{\prime}\right)=-\frac{e^{-\frac{2 \lambda_{1} \lambda_{2} t^{\prime}}{\lambda_{1}+\lambda_{2}}}}{2} \frac{\partial}{\partial x^{\prime}} I_{0}\left(\frac{\lambda_{1}+\lambda_{2}}{c_{1}+c_{2}} \sqrt{\frac{4\left(c_{1}+c_{2}\right)^{2} \lambda_{1}^{2} \lambda_{2}^{2} t^{\prime 2}}{\left(\lambda_{1}+\lambda_{2}\right)^{4}}-x^{\prime 2}}\right)
$$

We now present what we consider the most important result in this paper.

Theorem 4.1: The distribution of the position of the particle in the frame $(x, t)$ reads

$$
\begin{gathered}
p(x, t)=\frac{e^{-\frac{\left(\lambda_{1}+\lambda_{2}\right) t}{2}+\frac{\lambda_{2}-\lambda_{1}}{c_{2}+c_{1}} x+\frac{\left(\lambda_{2}-\lambda_{1}\right)\left(c_{2}-c_{1}\right)}{2\left(c_{2}+c_{1}\right)} t}}{c_{1}+c_{2}} t \\
{\left[\frac{\lambda_{1}+\lambda_{2}}{2} I_{0}\left(\frac{2 \sqrt{\lambda_{1} \lambda_{2}}}{c_{2}+c_{1}} \sqrt{\left(x+c_{2} t\right)\left(c_{1} t-x\right)}\right)\right.} \\
+\frac{\partial}{\partial t} I_{0}\left(\frac{2 \sqrt{\lambda_{1} \lambda_{2}}}{c_{2}+c_{1}} \sqrt{\left(x+c_{2} t\right)\left(c_{1} t-x\right)}\right) \\
\left.-\frac{\left(c_{2}-c_{1}\right)}{2} \frac{\partial}{\partial x} I_{0}\left(\frac{2 \sqrt{\lambda_{1} \lambda_{2}}}{c_{2}+c_{1}} \sqrt{\left(x+c_{2} t\right)\left(c_{1} t-x\right)}\right)\right] \\
+\frac{1}{2} e^{-\lambda_{1} t} \delta\left(x-c_{1} t\right)+\frac{1}{2} e^{-\lambda_{2} t} \delta\left(x+c_{2} t\right)
\end{gathered}
$$

for $-c_{2} t \leq x \leq c_{1} t$.

Proof: Taking into account formula (3.2) we get that

$$
\frac{4\left(c_{1}+c_{2}\right)^{2} \lambda_{1}^{2} \lambda_{2}^{2} t^{\prime 2}}{\left(\lambda_{1}+\lambda_{2}\right)^{4}}-x^{\prime 2}=\frac{4 \lambda_{1} \lambda_{2}}{\left(\lambda_{1}+\lambda_{2}\right)^{2}}\left(x+c_{2} t\right)\left(c_{1} t-x\right) \text {. }
$$

Of fundamental importance is to obtain the connection between $\frac{\partial}{\partial t^{\prime}}$ and $\frac{\partial}{\partial x}, \frac{\partial}{\partial t}$. We first note that

$$
\frac{\partial}{\partial t^{\prime}}=\frac{\partial}{\partial t} \frac{d t}{d t^{\prime}}+\frac{\partial}{\partial x} \frac{d x}{d t^{\prime}}=\left(\frac{\partial}{\partial t}+\frac{\partial}{\partial x} \frac{d x}{d t}\right) \frac{d t}{d t^{\prime}}
$$


and thus

$$
\frac{d t^{\prime}}{d t} \frac{\partial}{\partial t^{\prime}}=\frac{\partial}{\partial t}+\frac{\partial}{\partial x} \frac{d x}{d t}
$$

By using Remark 3.3, from (4.5) we get:

$$
\left\{\begin{array}{c}
\frac{\lambda_{1}+\lambda_{2}}{2 \lambda_{2}} \frac{\partial}{\partial t^{\prime}}=\frac{\partial}{\partial t}+c_{1} \frac{\partial}{\partial x} \\
\frac{\lambda_{1}+\lambda_{2}}{2 \lambda_{1}} \frac{\partial}{\partial t^{\prime}}=\frac{\partial}{\partial t}-c_{2} \frac{\partial}{\partial x}
\end{array}\right.
$$

Summing the identities in (4.6) yields

$$
\frac{\left(\lambda_{1}+\lambda_{2}\right)^{2}}{2 \lambda_{1} \lambda_{2}} \frac{\partial}{\partial t^{\prime}}=2 \frac{\partial}{\partial t}+\left(c_{1}-c_{2}\right) \frac{\partial}{\partial x}
$$

which, together with (4.4), permits us to pass from the absolutely continuous component of (4.1) to that of (4.3).

For the transformation of the singular component we must bear in mind that

and

$$
\left\{\begin{array}{l}
x^{\prime}-c^{\prime} t^{\prime}=\frac{2 \lambda_{1} \lambda_{2}}{\lambda_{1}+\lambda_{2}}\left(x-c_{1} t\right) \\
x^{\prime}+c^{\prime} t^{\prime}=\frac{2 \lambda_{1} \lambda_{2}}{\lambda_{1}+\lambda_{2}}\left(x+c_{2} t\right)
\end{array}\right.
$$

$$
-\frac{2 \lambda_{1} \lambda_{2}}{\lambda_{1}+\lambda_{2}} t^{\prime}=\frac{\lambda_{2}-\lambda_{1}}{\left(c_{1}+c_{2}\right)} x-\frac{\lambda_{1}+\lambda_{2}}{2} t+\frac{\left(c_{2}-c_{1}\right)\left(\lambda_{2}-\lambda_{1}\right)}{\left(c_{2}+c_{1}\right)} \frac{t}{2} \text {. }
$$

Since the Dirac delta function is concentrated in $x=c_{1} t$ and $x=-c_{2} t$ we have

$$
\frac{e^{-\frac{2 \lambda_{1} \lambda_{2}}{\lambda_{1}+\lambda_{2}} t^{\prime}}}{2}\left\{\delta\left(x^{\prime}+c^{\prime} t^{\prime}\right)+\delta\left(x^{\prime}-c^{\prime} t^{\prime}\right)\right\}=\frac{e^{-\lambda_{1} t}}{2} \delta\left(x-c_{1} t\right)+\frac{e^{-\lambda_{2} t}}{2} \delta\left(x+c_{2} t\right) .
$$

This concludes the proof of Theorem 4.1.

Remark 4.1: On the basis of the same reasoning it is possible to obtain the expression of the flow function in $(x, t)$ from (4.2).

After some calculations we have that

$$
\frac{d x^{\prime}}{d t^{\prime}} \frac{d t^{\prime}}{d t} \frac{\partial}{\partial x^{\prime}}=\frac{\partial}{\partial t}+\frac{\partial}{\partial x} \frac{d x}{d t}
$$

In view of Remark 3.3 and formula (3.8) we get

$$
\left\{\begin{array}{c}
\left(c_{1}+c_{2}\right) \frac{\lambda_{1}}{\lambda_{1}+\lambda_{2}} \frac{\partial}{\partial x^{\prime}}=\frac{\partial}{\partial t}+c_{1} \frac{\partial}{\partial x} \\
-\left(c_{1}+c_{2}\right) \frac{\lambda_{2}}{\lambda_{1}+\lambda_{2}} \frac{\partial}{\partial x^{\prime}}=\frac{\partial}{\partial t}-c_{2} \frac{\partial}{\partial x} .
\end{array}\right.
$$

Subtracting the identities in (4.9) yields

$$
\frac{\partial}{\partial x^{\prime}}=\frac{\partial}{\partial x}
$$


and thus the flow function in the frame $(x, t)$ can be written as

$$
\begin{aligned}
w(x, t)= & -\frac{1}{2} e^{-\frac{\left(\lambda_{1}+\lambda_{2}\right) t}{2}+\frac{\lambda_{2}-\lambda_{1}}{c_{2}+c_{1}}+\frac{\left(\lambda_{2}-\lambda_{1}\right)\left(c_{2}-c_{1}\right)}{2\left(c_{2}+c_{1}\right)} t} \\
& \cdot \frac{\partial}{\partial x} I_{0}\left(\frac{2 \sqrt{\lambda_{1} \lambda_{2}}}{c_{2}+c_{1}} \sqrt{\left(x+c_{2} t\right)\left(c_{1} t-x\right)}\right)
\end{aligned}
$$

for $-c_{2} t<x<c_{1} t$.

From (4.3) and (4.10) it is easy to obtain the distributions

$$
f_{1}=\frac{p+w}{2} \text { and } f_{2}=\frac{p-w}{2} .
$$

For example, the density of $f_{1}$ reads

$$
\begin{aligned}
f_{1}(x, t)= & \frac{1}{2\left(c_{1}+c_{2}\right)} e^{-\frac{\left(\lambda_{1}+\lambda_{2}\right) t}{2}+\frac{\lambda_{2}-\lambda_{1}}{c_{2}+c_{1}} x+\frac{\left(\lambda_{2}-\lambda_{1}\right)\left(c_{2}-c_{1}\right)}{2\left(c_{2}+c_{1}\right)} t} t \\
& {\left[\frac{\lambda_{1}+\lambda_{2}}{2} I_{0}\left(\frac{2 \sqrt{\lambda_{1} \lambda_{2}}}{c_{1}+c_{2}} \sqrt{\left(x+c_{2} t\right)\left(c_{1} t-x\right)}\right)\right.} \\
& +\frac{\partial}{\partial t} I_{0}\left(\frac{2 \sqrt{\lambda_{1} \lambda_{2}}}{c_{2}+c_{1}} \sqrt{\left(x+c_{2} t\right)\left(c_{1} t-x\right)}\right) \\
& \left.-c_{2} \frac{\partial}{\partial x} I_{0}\left(\frac{2 \sqrt{\lambda_{1} \lambda_{2}}}{c_{2}+c_{1}} \sqrt{\left(x+c_{2} t\right)\left(c_{1} t-x\right)}\right)\right]
\end{aligned}
$$

for $-c_{2} t<t<c_{1} t$.

Remark 4.2: We note that formulas (4.3), (4.10) and (4.11) coincide with the well-known distributions when no drift is assumed $\left(c_{1}=c_{2}=c, \lambda_{1}=\lambda_{2}=\lambda\right)$. Even more important is the fact that

$$
\int_{-c_{2} t}^{c_{1} t} p(x, t) d x=1,
$$

whose verification involves intriguing, yet cumbersome, calculations. This will be done in the next section since the necessary formulas will be extracted from general ones.

It may appear strange that the distribution (4.3), in the special case where the asymmetry is due only to different rates $\lambda_{1} \neq \lambda_{2}, c_{1}=c_{2}=c$, is much simpler than in the case where $\lambda_{1}=\lambda_{2}=\lambda$, but $c_{1} \neq c_{2}$.

In effect, in this case, formula (4.3) reduces to

$$
\begin{gathered}
p(x, t)=\frac{e^{-\frac{\left(\lambda_{1}+\lambda_{2}\right) t}{2}+\frac{\lambda_{2}-\lambda_{1}}{2 c} x}\left[\frac{\lambda_{1}+\lambda_{2}}{2} I_{0}\left(\frac{\sqrt{\lambda_{1} \lambda_{2}}}{c} \sqrt{c^{2} t^{2}-x^{2}}\right)\right.}{2 c} \\
\left.+\frac{\partial}{\partial t} I_{0}\left(\frac{\sqrt{\lambda_{1} \lambda_{2}}}{c} \sqrt{c^{2} t^{2}-x^{2}}\right)\right]
\end{gathered}
$$


for $|x|<c t$.

The reason for the simple structure of (4.12) is that the support of the distribution is symmetric and the asymmetry of the distribution is due only to the factor $\exp \frac{\lambda_{2}-\lambda_{1}}{2 c} x$.

\section{Derivation of the Distribution $P(x, t)$ by Solving an Initial-Value Problem}

The classical approach based on Fourier transforms permits us to obtain the characteristic function

$$
F(\beta, t)=\int_{-\infty}^{+\infty} e^{i \beta x} d P(x, t)
$$

of the distribution

$$
P(x, t)=P\{X(t) \leq x\}
$$

Theorem 5.1: The characteristic function of the distribution (5.2) is

$$
\begin{gathered}
F(\beta, t)=\frac{1}{2} e^{-\frac{1}{2}\left\{i \beta\left(c_{2}-c_{1}\right)+\left(\lambda_{1}+\lambda_{2}\right)\right\} t} \\
\cdot\left(1+\frac{\lambda_{1}+\lambda_{2}}{\sqrt{\left(\lambda_{1}+\lambda_{2}\right)^{2}-\beta^{2}\left(c_{1}+c_{2}\right)^{2}+2 i \beta\left(c_{1}+c_{2}\right)\left(\lambda_{2}-\lambda_{1}\right)}}\right) \\
+\left(1-\frac{\lambda_{1}+\lambda_{2}}{\sqrt{\left(\lambda_{1}+\lambda_{2}\right)^{2}-\beta^{2}\left(c_{1}+c_{2}\right)^{2}+2 i \beta\left(c_{1}+c_{2}\right)\left(\lambda_{2}-\lambda_{1}\right)}}\right) \\
\left.\cdot e^{-\frac{t}{2} \sqrt{\left(\lambda_{1}+\lambda_{2}\right)^{2}-\beta^{2}\left(c_{1}+c_{2}\right)^{2}+2 i \beta\left(c_{2}+c_{1}\right)\left(\lambda_{2}-\lambda_{1}\right)}}\right)
\end{gathered}
$$

for $\beta \in \mathbb{R}$ and $t \geq 0$.

Proof: We first note that the Fourier transform of equation (2.4) is

$$
\begin{gathered}
\frac{d^{2} F}{d t^{2}}+\left\{i \beta\left(c_{2}-c_{1}\right)+\left(\lambda_{2}+\lambda_{1}\right)\right\} \frac{d F}{d t} \\
+\left\{\frac{i \beta}{2}\left[\left(c_{2}-c_{1}\right)\left(\lambda_{2}+\lambda_{1}\right)-\left(\lambda_{2}-\lambda_{1}\right)\left(c_{2}+c_{1}\right)\right]+\beta^{2} c_{1} c_{2}\right\}=0 .
\end{gathered}
$$

It is straightforward that the general solution of (5.4) reads

$$
F(\beta, t)=e^{-\frac{t}{2}\left[i \beta\left(c_{2}-c_{1}\right)+\left(\lambda_{1}+\lambda_{2}\right)\right]}
$$




$$
\begin{gathered}
\cdot\left[H e^{\frac{t}{2} \sqrt{\left(\lambda_{1}+\lambda_{2}\right)^{2}-\beta^{2}\left(c_{1}+c_{2}\right)^{2}+2 i \beta\left(c_{2}+c_{1}\right)\left(\lambda_{2}-\lambda_{1}\right)}}\right. \\
\left.+K e^{-\frac{t}{2} \sqrt{\left(\lambda_{1}+\lambda_{2}\right)^{2}-\beta^{2}\left(c_{1}+c_{2}\right)^{2}+2 i \beta\left(c_{2}+c_{1}\right)\left(\lambda_{2}-\lambda_{1}\right)}}\right] .
\end{gathered}
$$

The constants $H$ and $K$ are evaluated using the fact that $F$ must satisfy the following initial conditions:

$$
\left\{\begin{array}{c}
F(\beta, 0)=1 \\
\left.\frac{d F}{d t}(\beta, t)\right|_{t=0}=\frac{1}{2} i \beta\left(c_{2}-c_{1}\right) .
\end{array}\right.
$$

While the first condition immediately follows from the fact that $p(x, 0)=\delta(x)$, the second one involves much more analysis.

The features of motion described in Section 2 authorize us to write

$$
\begin{gathered}
E e^{i \beta X(\Delta t)}=\frac{1}{2} e^{-i c_{2} \Delta t}\left(1-\lambda_{2} \Delta t\right)+\frac{1}{2} e^{i c_{1} \Delta t}\left(1-\lambda_{1} \Delta t\right) \\
+\frac{\left(\lambda_{1}+\lambda_{2}\right) \Delta t}{\left(c_{1}+c_{2}\right) \Delta t} \frac{1}{2} \int_{-c_{2} \Delta t}^{c_{1} \Delta t} e^{i \beta y} d y+o(\Delta t)
\end{gathered}
$$

since, in a small time elapse $[0, \Delta t$ ), either no velocity change occurs (and the particle, at time $\Delta t$, is at either endpoint of the interval $\left[-c_{2} \Delta t, c_{1} \Delta t\right]$ ) or one Poisson event happens (and the particle is then uniformly distributed inside that interval).

From (5.7) and some calculations, we get

$$
E e^{i \beta X(\Delta t)}=1+\frac{i \beta}{2}\left(c_{2}-c_{1}\right) \Delta t+o(\Delta t)
$$

and thus

$$
\left.\frac{d F}{d t}\right|_{t=0}=\lim _{\Delta t \rightarrow 0} \frac{E e^{i \beta X(\Delta t)}-1}{\Delta t}=\frac{i \beta}{2}\left(c_{2}-c_{1}\right)
$$

as claimed in (5.6).

A little algebra permits us to calculate $H$ and $K$ and thus obtain (5.3).

For the inversion of the characteristic function, we need three integrals which can be inferred from the relationship

$$
\int_{-c t}^{c t} e^{i \gamma x} I_{0}\left(\frac{\lambda}{c} \sqrt{c^{2} t^{2}-x^{2}}\right) d x=c \frac{\left\{e^{\frac{t}{2} \sqrt{\lambda^{2}-c^{2} \gamma^{2}}}-e^{-\frac{t}{2} \sqrt{\lambda^{2}-c^{2} \gamma^{2}}}\right\}}{\sqrt{\lambda^{2}-c^{2} \gamma^{2}}}
$$

obtained in [9].

For the sake of simplicity, we write

$$
A=\sqrt{\left(\lambda_{1}+\lambda_{2}\right)^{2}-\beta^{2}\left(c_{1}+c_{2}\right)^{2}+2 i \beta\left(c_{2}+c_{1}\right)\left(\lambda_{2}-\lambda_{1}\right)} .
$$

The formulas we must apply are 


$$
\begin{aligned}
& \int_{-c_{2} t}^{c_{1} t} e^{i \beta x} e^{\frac{\lambda_{2}-\lambda_{1}}{c_{2}+c_{1}} x} I_{0}\left(\frac{2 \sqrt{\lambda_{1} \lambda_{2}}}{c_{1}+c_{2}} \sqrt{\left(x+c_{2} t\right)\left(c_{1} t-x\right)}\right) d x \\
& =\left(c_{1}+c_{2}\right) e^{-i \beta\left(c_{2}-c_{1}\right) \frac{t}{2}-\frac{\left(\lambda_{2}-\lambda_{1}\right)\left(c_{2}-c_{1}\right)}{2\left(c_{2}+c_{1}\right)} t}\left\{\frac{e^{\frac{t}{2} A}-e^{-\frac{t}{2} A}}{A}\right\} \\
& \int_{-c_{2} t}^{c_{1} t} e^{i \beta x} e^{\frac{\lambda_{2}-\lambda_{1}}{c_{2}+c_{1}} x} \frac{\partial I_{0}}{\partial t}\left(\frac{2 \sqrt{\lambda_{1} \lambda_{2}}}{c_{1}+c_{2}} \sqrt{\left(x+c_{2} t\right)\left(c_{1} t-x\right)}\right) d x \\
& =\left(c_{1}+c_{2}\right)\left\{-\frac{i \beta\left(c_{2}-c_{1}\right)}{2}-\frac{\left(\lambda_{2}-\lambda_{1}\right)\left(c_{2}-c_{1}\right)}{2\left(c_{2}+c_{1}\right)}\right\} e^{-\frac{i \beta\left(c_{2}-c_{1}\right) t}{2}-\frac{\left(\lambda_{2}-\lambda_{1}\right)\left(c_{2}-c_{1}\right)}{2\left(c_{2}+c_{1}\right)} t} \\
& \cdot\left\{\frac{e^{\frac{t}{2} A}-e^{-\frac{t}{2} A}}{A}\right\}+\frac{c_{1}+c_{2}}{2} e^{-\frac{i \beta\left(c_{2}-c_{1}\right) t}{2}-\frac{\left(\lambda_{2}-\lambda_{1}\right)\left(c_{2}-c_{1}\right) t}{2\left(c_{2}+c_{1}\right)}}\left\{e^{\frac{t}{2} A}+e^{\left.-\frac{t}{2} A\right\}}\right. \\
& -c_{1} e^{i \beta c_{1} t} e^{\frac{\lambda_{2}-\lambda_{1}}{c_{2}+c_{1}} c_{1} t}-c_{2} e^{-i \beta c_{2} t^{-\frac{\lambda_{2}-\lambda_{1}}{c_{2}+c_{1}} c_{2} t} .}
\end{aligned}
$$

The third formula we need is

$$
\begin{gathered}
\int_{-c_{2} t}^{c_{1} t} e^{i \beta x} e^{\frac{\lambda_{2}-\lambda_{1}}{c_{2}+c_{1}} x} \frac{\partial I_{0}}{\partial x}\left(\frac{2 \sqrt{\lambda_{1} \lambda_{2}}}{c_{1}+c_{2}} \sqrt{\left(x+c_{2} t\right)\left(c_{1} t-x\right)}\right) d x \\
=e^{i \beta c_{1} t} e^{\frac{\lambda_{2}-\lambda_{1}}{c_{2}+c_{1}} c_{1} t}-e^{-i \beta c_{2} t} e^{-\frac{\lambda_{2}-\lambda_{1}}{c_{2}+c_{1}} c_{2} t} \\
-\left(c_{1}+c_{2}\right)\left\{i \beta+\frac{\lambda_{2}-\lambda_{1}}{c_{2}+c_{1}}\right\} e^{-i \beta\left(c_{2}-c_{1}\right) \frac{t}{2}-\frac{\left(\lambda_{2}-\lambda_{1}\right)\left(c_{2}-c_{1}\right)}{2\left(c_{2}+c_{1}\right)} t}\left\{\frac{e^{\frac{t}{2} A}-e^{-\frac{t}{2} A}}{A}\right\}
\end{gathered}
$$

Formulas (5.11) and (5.12) are closely connected by

$$
\begin{aligned}
& \int_{-c_{2} t}^{c_{1} t} e^{i \beta x} e^{\frac{\lambda_{2}-\lambda_{1}}{c_{2}+c_{1}} x} \frac{\partial I_{0}}{\partial t}\left(\frac{2 \sqrt{\lambda_{1} \lambda_{2}}}{c_{1}+c_{2}} \sqrt{\left(x+c_{2} t\right)\left(c_{1} t-x\right)}\right) d x \\
& =\frac{c_{2}-c_{1}}{2}\left\{\int_{-c_{2} t}^{c_{1} t} e^{i \beta x} e^{\frac{\lambda_{2}-\lambda_{1}}{c_{2}+c_{1}} x} \frac{\partial I_{0}}{\partial x}\left(\frac{2 \sqrt{\lambda_{1} \lambda_{2}}}{c_{1}+c_{2}} \sqrt{\left(x+c_{2} t\right)\left(c_{1} t-x\right)}\right) d x\right. \\
& \left.-e^{i \beta c_{1} t} e^{\frac{\lambda_{2}-\lambda_{1}}{c_{2}+c_{1}} c_{1} t}+e^{-i \beta c_{2} t} e^{-\frac{\lambda_{2}-\lambda_{1}}{c_{2}+c_{1}} c_{2} t}\right\}
\end{aligned}
$$




$$
\begin{gathered}
+\frac{c_{1}+c_{2}}{2} e^{-\frac{i \beta\left(c_{2}-c_{1}\right)}{2} t-\frac{\left(\lambda_{2}-\lambda_{1}\right)\left(c_{2}-c_{1}\right)}{2\left(c_{2}+c_{1}\right)} t}\left\{e^{\frac{t}{2} A}+e^{\left.-\frac{t}{2} A\right\}}\right. \\
-c_{1} e^{i \beta c_{1} t} e^{\frac{\lambda_{2}-\lambda_{1}}{c_{2}+c_{1}} c_{1} t}-c_{2} e^{-i \beta c_{2} t} e^{-\frac{\lambda_{2}-\lambda_{1}}{c_{2}+c_{1}} c_{2} t}
\end{gathered}
$$

as a simple comparison between (5.11) and (5.12) shows.

With this at hand, the characteristic function (5.3) can be written as:

$$
\begin{gathered}
F(\beta, t)=\frac{e^{-\frac{\left(\lambda_{1}+\lambda_{2}\right) t}{2}}}{2}\left\{e^{-\frac{i \beta\left(c_{2}-c_{1}\right)}{2} t\left(e^{\frac{t}{2} A}+e^{\left.-\frac{t}{2} A\right)}\right.}\right. \\
\left.+\left(\lambda_{1}+\lambda_{2}\right) e^{-\frac{i \beta\left(c_{2}-c_{1}\right)}{2} t}\left(\frac{e^{\frac{t}{2} A}-e^{-\frac{t}{2} A}}{A}\right)\right\}
\end{gathered}
$$

(using (5.13) for the first term and (5.10) for the second one)

$$
\begin{aligned}
& =\frac{e^{-\frac{\left(\lambda_{1}+\lambda_{2}\right) t}{2}}}{2} e^{\frac{\left(\lambda_{2}-\lambda_{1}\right)\left(c_{2}-c_{1}\right)}{2\left(c_{2}+c_{1}\right)} t} \\
& {\left[\frac{\lambda_{1}+\lambda_{2}}{c_{1}+c_{2}} \int_{-c_{2} t}^{c_{1} t} e^{i \beta x} e^{\frac{\lambda_{2}-\lambda_{1}}{c_{2}+c_{1}} x} I_{0}\left(\frac{2 \sqrt{\lambda_{1} \lambda_{2}}}{c_{1}+c_{2}} \sqrt{\left(x+c_{2} t\right)\left(c_{1} t-x\right)}\right) d x\right.} \\
& +\frac{2}{c_{1}+c_{2}} \int_{-c_{2} t}^{c_{1} t} e^{i \beta x} e^{\frac{\lambda_{2}-\lambda_{1}}{c_{2}+c_{1}} x} \frac{\partial I_{0}}{\partial t}\left(\frac{2 \sqrt{\lambda_{1} \lambda_{2}}}{c_{1}+c_{2}} \sqrt{\left(x+c_{2} t\right)\left(c_{1} t-x\right)}\right) d x \\
& -\frac{c_{2}-c_{1}}{c_{1}+c_{2}} \int_{-c_{2} t}^{c_{1} t} e^{i \beta x} e^{\frac{\lambda_{2}-\lambda_{1}}{c_{2}+c_{1}} x} \frac{\partial I_{0}}{\partial x}\left(\frac{2 \sqrt{\lambda_{1} \lambda_{2}}}{c_{1}+c_{2}} \sqrt{\left(x+c_{2} t\right)\left(c_{1} t-x\right)}\right) d x \\
& +\frac{c_{2}-c_{1}}{c_{1}+c_{2}} e^{i \beta c_{1} t} e^{\frac{\lambda_{2}-\lambda_{1}}{c_{2}+c_{1}} c_{1} t}-\frac{c_{2}-c_{1}}{c_{1}+c_{2}} e^{-i \beta c_{2} t} e^{-\frac{\lambda_{2}-\lambda_{1}}{c_{2}+c_{1}} c_{2} t} \\
& \left.+\frac{2 c_{1}}{c_{1}+c_{2}} e^{i \beta c_{1} t} e^{\frac{\lambda_{2}-\lambda_{1}}{c_{2}+c_{1}} c_{1} t}+\frac{2 c_{2}}{c_{1}+c_{2}} e^{-\beta c_{2} t} e^{-\frac{\lambda_{2}-\lambda_{1}}{c_{2}+c_{1}} c_{2} t}\right] \\
& =\frac{e^{-\frac{\left(\lambda_{2}+\lambda_{1}\right) t}{2}+\frac{\left(\lambda_{2}-\lambda_{1}\right)\left(c_{2}-c_{1}\right)}{2\left(c_{2}+c_{1}\right)} t}}{c_{1}+c_{2}} \\
& \cdot\left[\frac{\lambda_{1}+\lambda_{2}}{2} \int_{-c_{2} t}^{c_{1} t} e^{i \beta x} e^{\frac{\lambda_{2}-\lambda_{1}}{c_{2}+c_{1}} x} I_{0}\left(\frac{2 \sqrt{\lambda_{1} \lambda_{2}}}{c_{1}+c_{2}} \sqrt{\left(x+c_{2} t\right)\left(c_{1} t-x\right)}\right) d x\right.
\end{aligned}
$$


24

L. BEGHIN, L. NIEDDU and E. ORSINGHER

$$
\begin{gathered}
+\int_{-c_{2} t}^{c_{1} t} e^{i \beta x} e^{\frac{\lambda_{2}-\lambda_{1}}{c_{2}+c_{1}} x} \frac{\partial I_{0}}{\partial t}\left(\frac{2 \sqrt{\lambda_{1} \lambda_{2}}}{c_{1}+c_{2}} \sqrt{\left(x+c_{2} t\right)\left(c_{1} t-x\right)}\right) d x \\
\left.-\frac{c_{2}-c_{1}}{2} \int_{-c_{2} t}^{c_{1} t} e^{i \beta x} e^{\frac{\lambda_{2}-\lambda_{1}}{c_{2}+c_{1}} x} \frac{\partial I_{0}}{\partial x}\left(\frac{2 \sqrt{\lambda_{1} \lambda_{2}}}{c_{1}+c_{2}} \sqrt{\left(x+c_{2} t\right)\left(c_{1} t-x\right)}\right) d x\right] \\
+\frac{1}{2} e^{-\lambda_{1} t} e^{i \beta c_{1} t}+\frac{1}{2} e^{-\lambda_{2} t} e^{-i \beta c_{2} t} .
\end{gathered}
$$

From this we immediately obtain again the distribution (4.3).

Remark 5.1: In order to prove that the distribution (4.3) integrates to one, we only have to integrate the absolutely continuous part and use formulas $(5.10),(5.11)$ and (5.12) when $\beta=0$. We present some details here

$$
\begin{aligned}
& \operatorname{Pr}\left\{-c_{2} t<X(t)<c_{1} t\right\}=\frac{e^{-\frac{\left(\lambda_{2}+\lambda_{1}\right) t}{2}+\frac{\left(\lambda_{2}-\lambda_{1}\right)\left(c_{2}-c_{1}\right)}{2\left(c_{2}+c_{1}\right)} t}}{c_{1}+c_{2}} \\
& \cdot\left[\frac{\lambda_{1}+\lambda_{2}}{2} \int_{-c_{2} t}^{c_{1} t} \frac{\lambda_{2}-\lambda_{1}}{c^{c_{2}+c_{1}} x} I_{0}\left(\frac{2 \sqrt{\lambda_{1} \lambda_{2}}}{c_{1}+c_{2}} \sqrt{\left(x+c_{2} t\right)\left(c_{1} t-x\right)}\right) d x\right. \\
& +\int_{-c_{2} t}^{c_{1} t} \frac{\lambda_{2}-\lambda_{1}}{c_{2}+c_{1}} x \frac{\partial I_{0}}{\partial t}\left(\frac{2 \sqrt{\lambda_{1} \lambda_{2}}}{c_{1}+c_{2}} \sqrt{\left(x+c_{2} t\right)\left(c_{1} t-x\right)}\right) d x \\
& \left.-\frac{c_{2}-c_{1}}{2} \int_{-c_{2} t}^{c_{1} t} e^{\frac{\lambda_{2}-\lambda_{1}}{c_{2}+c_{1}} x} \frac{\partial I_{0}}{\partial x}\left(\frac{2 \sqrt{\lambda_{1} \lambda_{2}}}{c_{1}+c_{2}} \sqrt{\left(x+c_{2} t\right)\left(c_{1} t-x\right)}\right) d x\right] \\
& =\frac{e^{-\frac{\left(\lambda_{2}+\lambda_{1}\right)}{2} t}}{c_{1}+c_{2}}\left[\frac { \lambda _ { 1 } + \lambda _ { 2 } } { 2 ( \lambda _ { 1 } + \lambda _ { 2 } ) } ( c _ { 1 } + c _ { 2 } ) \left(e^{\frac{t}{2}\left(\lambda_{1}+\lambda_{2}\right)}-e^{\left.-\frac{t}{2}\left(\lambda_{1}+\lambda_{2}\right)\right)}\right.\right. \\
& +\frac{c_{1}+c_{2}}{\lambda_{1}+\lambda_{2}}\left\{-\frac{\left(\lambda_{2}-\lambda_{1}\right)\left(c_{2}-c_{1}\right)}{2\left(c_{1}+c_{2}\right)}\right\}\left\{e^{\frac{t}{2}\left(\lambda_{1}+\lambda_{2}\right)}-e^{-\frac{t}{2}\left(\lambda_{1}+\lambda_{2}\right)}\right\} \\
& +\frac{c_{1}+c_{2}}{2}\left(e^{\frac{t}{2}\left(\lambda_{1}+\lambda_{2}\right)}+e^{-\frac{t}{2}\left(\lambda_{1}+\lambda_{2}\right)}\right)-c_{1} e^{\frac{\lambda_{2}-\lambda_{1}}{c_{2}+c_{1}} c_{1} t}-c_{2} e^{-\frac{\lambda_{2}-\lambda_{1}}{c_{2}+c_{1}} c_{2} t} \\
& +\frac{c_{2}-c_{1}}{2} \frac{c_{1}+c_{2}}{\lambda_{1}+\lambda_{2}} \frac{\lambda_{2}-\lambda_{1}}{c_{2}+c_{1}}\left(e^{\frac{t}{2}\left(\lambda_{1}+\lambda_{2}\right)}-e^{-\frac{t}{2}\left(\lambda_{1}+\lambda_{2}\right)}\right)
\end{aligned}
$$




$$
\left.-\frac{c_{2}-c_{1}}{2} e^{\frac{\lambda_{2}-\lambda_{1}}{c_{2}+c_{1}} c_{1} t}+\frac{c_{2}-c_{1}}{2} e^{-\frac{\lambda_{2}-\lambda_{1}}{c_{2}+c_{1}} c_{2} t}\right]
$$

(observe that coefficients of $e^{-\frac{\lambda_{2}+\lambda_{1}}{2} t}$ cancel throughout)

$$
\begin{gathered}
=\frac{e^{-\frac{\left(\lambda_{2}+\lambda_{1}\right) t}{2}}}{c_{1}+c_{2}}\left[\left(c_{2}+c_{1}\right) e^{\frac{t}{2}\left(\lambda_{1}+\lambda_{2}\right)}-\frac{c_{2}+c_{1}}{2} e^{\frac{\lambda_{2}-\lambda_{1}}{2} t}-\frac{c_{2}+c_{1}}{2} e^{-\frac{\lambda_{2}-\lambda_{1}}{2} t}\right] \\
=1-\frac{1}{2} e^{-\lambda_{1} t}-\frac{1}{2} e^{-\lambda_{2} t}=1-P\left\{X(t)=+c_{1} t\right\}-P\left\{X(t)=-c_{2} t\right\} .
\end{gathered}
$$

\section{References}

[1] Cane, V., Diffusion models with relativity effects, In: Perspectives in Probability and Statistics (ed. by Gani), Academic Press for the Applied Probability Trust, Sheffield, UK (1975), 263-273.

[2] Foong, S.K., First-passage time, maximum displacement and Kac's solution of the telegrapher equation, Phys. Rev. A 46 (1992), 707-710.

[3] Foong, S.K. and Kanno, S., Properties of the telegrapher's random process with or without a trap, Stoch. Proc. and their Appl. (1994), 147-173.

[4] Kabanov, Yu.M., On the probabilistic representation of the telegraph equation, Theory of Prob. and its Appl. 37 (1992), 425-426.

[5] Kolesnik, A.D., The equations of Markovian random evolution on the line, J. of Appl. Prob. 35 (1998), 27-35.

[6] Orsingher, E., Hyperbolic equations arising in random models, Stoch. Proc. and their Appl. (1985), 49-66.

[7] Orsingher, E., Probabilistic models connected with wave equations, Boll. Un. Mate. Italiana 7:1-B (1987), 423-438.

[8] Orsingher, E., Probability law, flow function, maximum distribution of wavegoverned random motions and their connections with Kirchhoff's laws, Stoch. Proc. and their Appl. (1990), 49-66.

[9] Orsingher, E., Motions with reflecting and absorbing barriers driven by the telegraph equation, Random Oper. and Stoch. Eqns. 3:1 (1995), 9-21. 


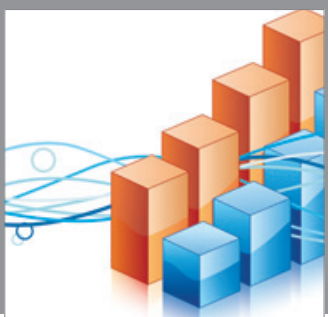

Advances in

Operations Research

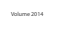

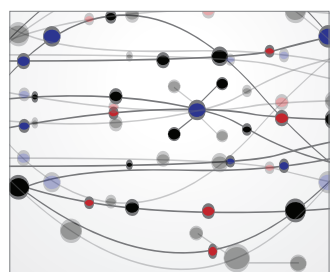

\section{The Scientific} World Journal
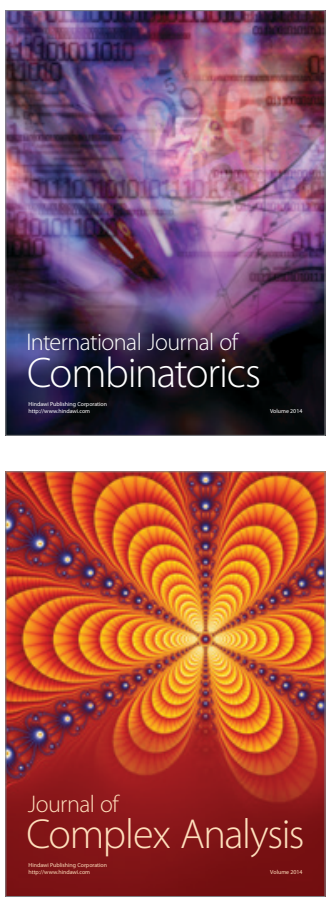

International Journal of

Mathematics and

Mathematical

Sciences
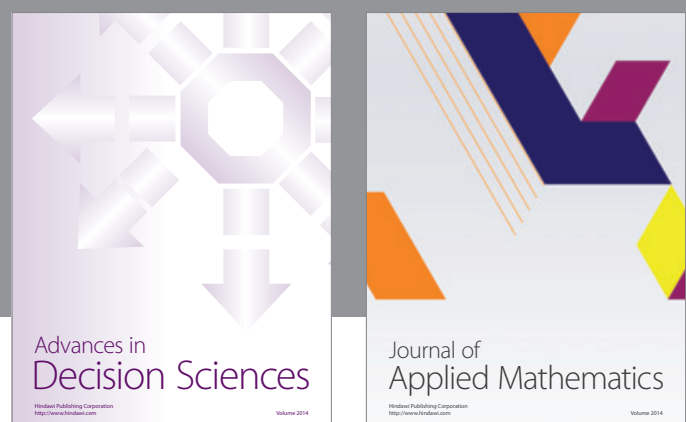

Journal of

Applied Mathematics
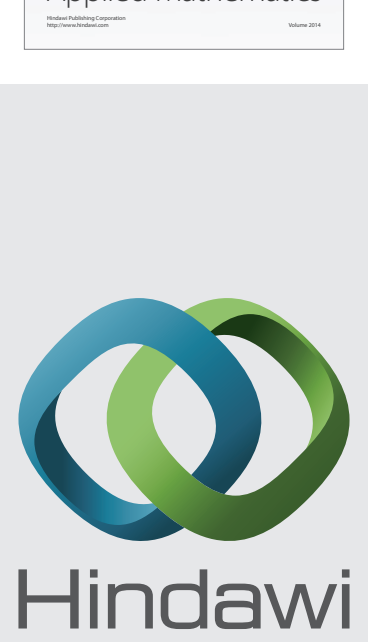

Submit your manuscripts at http://www.hindawi.com
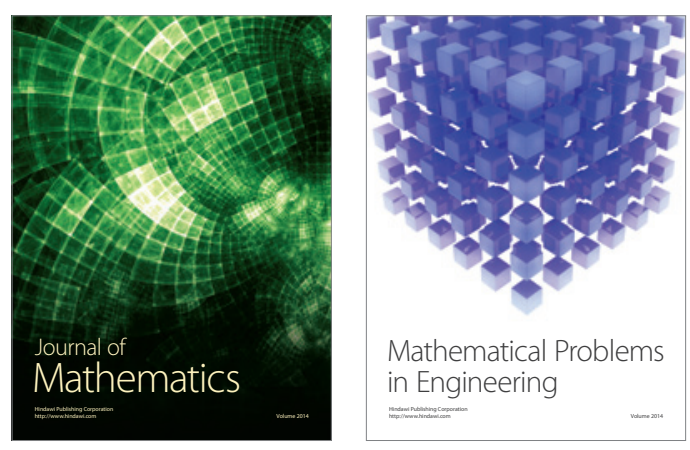

Mathematical Problems in Engineering
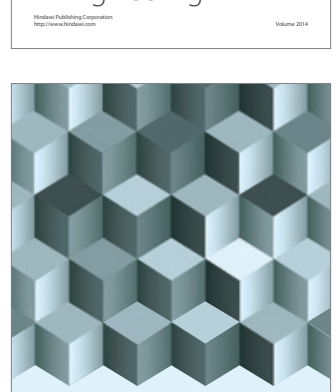

Journal of

Function Spaces
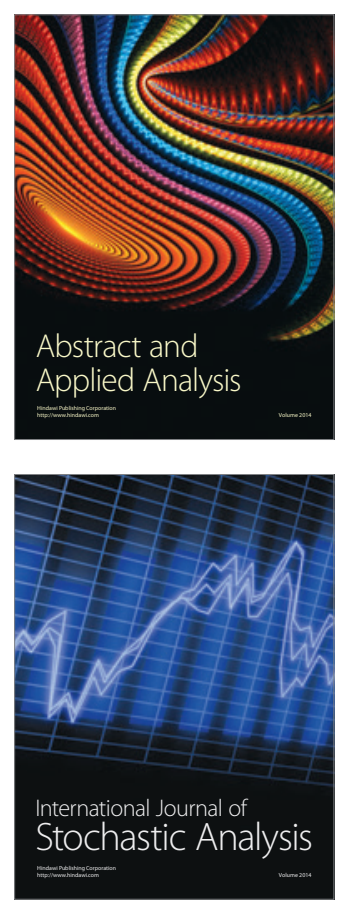

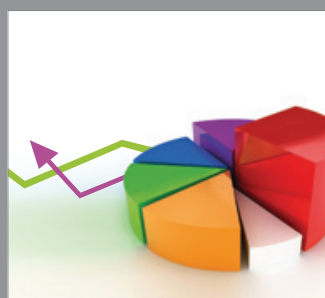

ournal of

Probability and Statistics

Promensencen
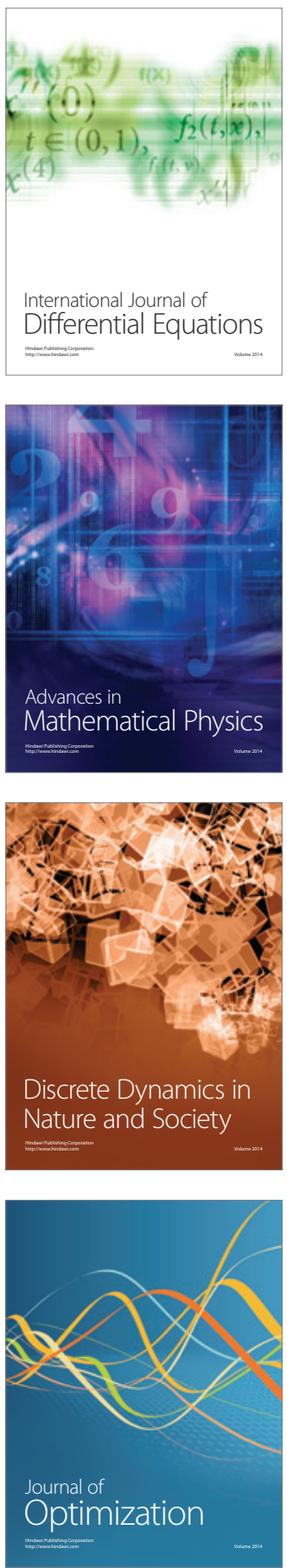